Internationalisation, modernisation et régionalisation - L'orientation du développement de l'enseignement des médias dans un nouveau siècle

\title{
Yuexin Qiang
}

\section{OpenEdition}

\section{Journals}

Édition électronique

URL : http://journals.openedition.org/communicationorganisation/2937

DOI : 10.4000/communicationorganisation.2937

ISSN : 1775-3546

Éditeur

Presses universitaires de Bordeaux

Édition imprimée

Date de publication : 1 mai 2004

ISSN : 1168-5549

Référence électronique

Yuexin Qiang, «Internationalisation, modernisation et régionalisation - L'orientation du

développement de l'enseignement des médias dans un nouveau siècle », Communication et organisation [En ligne], 24 | 2004, mis en ligne le 27 mars 2012, consulté le 20 avril 2019. URL : http:// journals.openedition.org/communicationorganisation/2937 ; DOI : 10.4000/ communicationorganisation.2937

Ce document a été généré automatiquement le 20 avril 2019.

(C) Presses universitaires de Bordeaux 


\title{
Internationalisation, modernisation et régionalisation - L'orientation du développement de l'enseignement des médias dans un nouveau siècle
}

\author{
Yuexin Qiang
}

1 Notre pays n'a connu les médias qu'après une longue période d'isolement du monde extérieur due à des problèmes historiques. En 1956. à l'intérieur du pays, il n'y avait que très peu de personnes qui connaissaient ce mot : en juillet 1978. Le document sur la presse étrangère de l'Université Fudan. qui se situe à Shanghai, a introduit pour la première fois la notion de média (la traduction adjoignait l'adjectif public au nom média: média public) : en octobre 1978. Neichuan Fangmei. le président de l'Association de la Presse japonaise est venu en Chine, il a introduit l'expression « mass media » dans son discours : le mot a beaucoup étonné les chercheurs chinois du domaine: en avril et mai 1982. Wilbert Slam, chercheur américain faisant autorité, accompagné par son élève Yu Yelu. a visité la Chine. C'est à ce moment-là enfin, que la notion de média a été vraiment connue en Chine. Dès lors, notre milieu journalistique commença à apprendre et à introduire le média avec zèle, à la fois avec sincérité et anxiété. Et à la dernière décade de novembre 1982 la première conférence nationale sur les médias a eu lieu à l'institut des recherches de presse de CAS (Académie Chinoise des Sciences). En seulement quelques décennies, notre pays a fait de grands progrès dans la connaissance du domaine des médias, et plusieurs établissements de renseignement supérieur ont érigé l'étude des médias en discipline, Grâce à leur effort inlassable, on améliore beaucoup le contenu et la méthode de l'éducation aux médias, la façon de former les jeunes, etc. Particulièrement grâce à la politique d'ouverture, l'éducation des médias de notre pays a renforcé la communication avec des pays d'outre-mer et les régions Taïwan et Hongkong: cela permet, dans le cadre des cours d'éducation aux médius de notre pays, d'apprendre de nouvelles connaissances au niveau le plus avancé du monde et cela réduit progressivement la distance entre notre pays et les pays développés dans ce domaine, lit 
les personnalités formées par notre éducation aux médias ont manifesté une certaine force, en concurrence avec des confrères internationaux.

\section{L'internationalisation, une tendance irréversible}

2 La réelle internationalisation de renseignement supérieur au sens moderne débute au XIX ${ }^{\mathrm{e}}$ siècle, et se développe au $\mathrm{XX}^{\mathrm{e}}$ siècle, il y a un développement énorme, en particulier après la deuxième guerre mondiale, et aujourd'hui, elle est totale, y compris pour renseignement des médias.

\section{Connotation de l'internationalisation de l'enseignement des médias}

L'internationalisation de renseignement des médias est un procès graduel et continu, il faut que notre enseignement envisage le monde extérieur, attache l'importance aux recherches et forme des talents capables de participer à la compétition internationale, en prenant les expériences des instituts frères par le biais des vaste communications et coopérations internationales. Quant.au contenu. il concerne deux aspects: l'internationalisation de l'enseignement et celle de la gestion. Et la première est composée par l'internationalisation des éléments de la formation (objectifs de la formation, contenu des enseignements, méthodes et moyens pédagogiques, etc.) des groupes de professeurs, des gestionnaires, des étudiants, des établissements. Or pour vraiment et effectivement réaliser l'internationalisation de l'enseignement, il lui faut l'internationalisation de la gestion d'enseignement.

\section{La voie d'internationalisation de l'enseignement des médias}

4 1. Renforcer l'éducation et les formations des langues étrangères. Bien que nous avions déjà développé l'étude du média national, il reste quand même un problème : la plupart des ouvrages traitant des médias viennent des pays étrangers, c'est pourquoi on doit renforcer l'enseignement et les formations en langues étrangères. Ici. j'insiste non seulement sur l'augmentation quantitative, mais surtout sur la réforme de «modèle d'examen » qui existe depuis longtemps dans notre éducation traditionnelle. Donc, on doit aussi organiser des formations pour les professeurs et les personnes de gestion, et développer le savoir-faire de chacun en langue étrangère.

5 2. Perfectionner des cours internationaux. Les cours internationaux ont pour but la compréhension internationale, donc, les professeurs doivent former le sens international des élèves. L'enseignement des médias est venu des pays étrangers, et aujourd'hui, les êtres humains vivent ensemble dans « un village global ». la mondialisation se développe à grande vitesse : personne ne peut se tenir éloigné de l'environnement international.

6 3. Renforcer la communication internationale des professeurs et des élèves. D'abord, on doit élargir le contact et la communication interuniversitaires: ensuite, on doit développer l'envoi des étudiants à l'étranger et aussi recruter des étudiants étrangers : enfin, on peut recruter des professeurs et des gestionnaires étrangers si les condition le permettent. Bien sûr. il existe des contraintes financières, mais dans la situation présente, l'université doit impérativement accélérer le développement de l'enseignement des 
médias par l'invitation de chercheurs reconnus afin qu'ils donnent des conférences et des cours.

7 4. Renforcer les coopérations internationales en matière de recherche. À une époque où les sciences et les technologies se développent énormément, devant des problèmes sociaux de plus en plus compliqués, on ne peut pas se contenter des recherches menées au niveau d'un seul pays ou d'une seule université. Il est bien nécessaire de mener des coopérations internationales.

8 5. Développer la coopération internationale d'enseignement. Développer la coopération internationale d'enseignement est un moyen efficace pour développer en profondeur l'internationalisation de renseignement supérieur et former des talents aptes à la compétition internationale. L'enseignement des médias de notre pays doit aussi accélérer la coopération internationale en matière d'enseignement, prendre les expériences des pays étrangers comme référence et perfectionner la qualité de la formation des jeunes.

\section{La modernisation, une poursuite inlassable}

\section{Le challenge de la modernisation}

9 En réalité, le fait que l'enseignement des médias traditionnel est frappe par le média moderne n'est pas un nouveau problème. Dès le siècle dernier, au moment où la radio et la télévision se généralisèrent successivement, le modèle traditionnel d'enseignement des médias se trouvait déjà devant un challenge sérieux. Évidemment, le «modèle enseignement de la presse » fondé à une époque où le journal prédominait ne correspond pas aux pratiques des médias électroniques qui sont la grande vitesse, l'étendue du domaine, la variété des domaines et la représentation figurative. De nombreuses expériences et leçons venues des pratiques des médias électroniques sont devenues une partie importante de renseignement des médias, lit. en seulement quelques décennies, la situation mondiale a connu encore une fois de grands changements: une morphologie sociale et économique tout à fait nouvelle se présente devant le monde, et future morphologie sociale qui remplacera la traditionnelle, une société informatique et intellectuelle se présente de plus en plus clair devant nous. Avec la complexification de forme des médias, l'enseignement supérieur des médias se trouve devant un problème difficile : comment faire face au choc et au challenge provoqués par les nouveaux médias dont le prototype est constitué par les autoroutes de l'information aussi bien qu'au choc et au challenge venus de l'entrée dans l'ère de "l'économie intellectuelle» qui se manifeste dans le marché des médias: au choc et au challenge qu'impose la nouvelle structure du cadre international des médias, etc.

\section{Modernisation de l'enseignement des médias à l'époque de l'informatique}

10 1. Modernisation de l'idée de l'enseignement. Après l'entrée à l'époque informatique, parce que l'Internet a changé les moyens des mass média, non seulement la presse traditionnelle et sa pratique sont déliées par lui mais aussi la théorie des mass média est mise en cause. Les médias et les théories de la presse anciens ne correspondent plus au média Internet. Un nouveau système de masse média est en train de développer, basé sur l'Internet. 
11 2. Modernisation du contenu de l'enseignement. Avec le développement des technologies des médias modernes et l'utilisation des nouvelles sciences et technologies dans ce domaine, avec l'augmentation continuelle des proportions technologiques dans la vie humaine, il faut briser l'ancien cadre intellectuel de division stricte et monotone de travail, en ce qui concerne les formes, les moyens et les contenus des médias. Il nous faut audacieusement encourager les étudiants à choisir des cours interdisciplinaires et franchir les limites de leur propre spécialité, il faut en particulier briser l'idée ancienne qui annexe la spécialité des médias à la faculté des lettres, et reconnaître le statut de presse-média dans la société informatique et l'industrie informatique futures. On accroît ainsi le rôle de la faculté des sciences et techniques dans le programme d'enseignement, surtout les sciences de pointe comme l'informatique et la technologie Internet. Lt réellement actualiser ce que les étudiants apprennent.

12 3. Modernisation des moyens d'enseignement. À l'époque de l'informatique, l'enseignement ne saurait se borner aux méthodes manuelles. L'utilisation des diverses technologies comme l'ordinateur, le multimédia, la communication Internet et la technologie digitale amène de nouveaux changements aux méthodes d'enseignement. La combinaison de différentes méthodes non seulement élargit l'horizon des étudiants, mais aussi les intéresse beaucoup et les mène à apprendre plus activement.

\section{La régionalisation, le centre inchangeable}

Dans l'éducation supérieure de notre pays, l'enseignement et les recherches ont toujours une relation étroite, donc, en parlant de l'enseignement des médias, on ne peut pas négliger le terme qui est très populaire dans le domaine de la recherche sur les médias : la régionalisation des médias. La première conférence nationale sur les médias de $19 \mathrm{X} 2 \mathrm{a}$ proposé le principe d'attitude par rapport au média occidental, en 16 caractères chinois qu'on peut traduire ainsi: "connaître systématiquement, étudier sérieusement, apprendre avec choix et créer indépendamment ». Dès lors, la régionalisation des média, ou bien « la sinoisation». est devenue la problématique central de nos recherches en la matière. Or aujourd'hui, au XXI ${ }^{e}$ siècle, on ne peut qu'exprimer un certain pessimisme face à la réalité: malgré son développement de vingt années en Chine, l'industrie des médias se trouve encore dans l'étape introductive de l'apprentissage, et ce travail reste encore à renforcer. L'état actuel est celui des recherches de type « compréhensif » tandis que les recherches "créatives" sont extrêmement rares. Bien qu'on ait fait aussi quelques recherches autonomes, la catégorie, les modèles et les méthodes de recherche sont tous venus de l'occident, et sont principalement de l'école traditionnelle (c'est-à-dire l'école empirique américaine). Les plus récents de nos principes restent encore une hypothèse. On en est encore à se demander : Quelles sont les causes de ce problème ? Estce qu'on doit régionaliser le média? Et comment? Ou bien est-ce la régionalisation des objets de recherche? Des méthodes de recherche ? Ou encore des théories?

D'abord, rappelons-nous un peu du processus d'amélioration de ce courant idéologique dans notre pays. Il est de notoriété publique que dès les années 1980 plusieurs conférences de recherches sur les médias ont contribué beaucoup au développement des médias sur le continent chinois. Dans ces conférences, les chercheurs discutent ensemble et présentent leurs opinions à propos du développement des médias. Une des problématiques est la régionalisation des médias. A propos de cette problématique, le milieu intellectuel lient toujours des opinions différentes, mais on accepte toutes les 
opinions que nos recherches peuvent ou doivent créer. Ici. on utilise seulement « régionalisation » pour souligner le sens de créativité.

En proposant le principe des 16 caractères chinois, la première conférence de 1982 laissait présager l'orientation de régionalisation des médias.

La deuxième conférence de 1986 a proposé précisément le but de " construire l'industrie des médias de spécialité chinoise " a fixé l'étude delà presse comme la direction principale des recherches des médias et a décidé de concentrer les forces pour écrire l'ouvrage de presse-média se rapportant à notre situation nationale.

17 La troisième et la quatrième, en 1993 et 1995 ont spécialement discuté le problème de la régionalisation. À ce moment-là, la régionalisation des médias est devenue un sujet majeur de recherche, disputé chaleureusement par le milieu intellectuel. Cette chaleur reflète l'importance que le milieu intellectuel attache à la régionalisation des médias et la profondeur de recherche sur les médias qui ne se borne pas à l'introduction et à l'évaluation des médias occidentaux.

Quant à cette problématique, une opinion est d'insister fermement sur la régionalisation des médias. Au contraire, une autre croit qu'on ne peut pas généraliser partout «la spécificité chinoise». et qu'il n'est pas nécessaire de coller l'étiquette chinoise à la recherche sur les médias, qu'il n'est pas convenable de mettre excessivement l'accent sur «la spécificité chinoise ». Il faut que la spécialité chinoise se manifeste naturellement en cours de recherche, lin tant que Chinois, nous considérons naturellement notre situation nationale cl notre réalité en étudiant le média, ainsi, la spécificité chinoise se manifeste-telle naturellement. Quant au média occidental, nous devons encore le connaître systématiquement et l'étudier profondément. Insister trop sur la spécificité chinoise peut avoir pour effet d'influencer l'assimilation sélective du média occidental. Cette opinion ne s'oppose pas à la régionalisation des médias, ce qui l'intéresse, c'est comment avoir une attitude correcte auprès du média occidental, autrement dit comment traiter et développer le média continental de la Chine. Cette opinion-là reflète l'attention que les chercheurs font au développement du média continental.

Enfin, le milieu des médius continental a perfectionné et unifié ses opinions à propos de la régionalisation: réaliser la régionalisation des médias et éviter des problèmes possibles.

20 Quant à savoir comment accélérer la régionalisation des médias, les chercheurs ont donné de nombreuses propositions, on les résume ci-dessous :

21 1/. Etudier systématiquement les écoles principales des médias étrangers, écarter les opinions incorrectes et les parties inutiles, prendre les expériences comme référence.

2/. Résumer sérieusement l'expérience, la pensée et la théorie de notre histoire des médias, et les étudier respectivement.

$233 /$. Enquêter sur l'état et les problèmes de notre industrie des médias, les étudier afin de connaître les points forts et faiblesses, puis réformer et agir audacieusement pour contribuer à établir un nouveau système de " presse-média ».

24 4/. Faire des recherches sur les problèmes des médias avec la réalité du socialisme primaire de la Chine pour établir la théorie des médias de spécialité chinoise.

25 La dispute sur la régionalisation stimule le sens créatif du milieu intellectuel, en plus, l'idée de créativité s'unifie progressivement: c'est bien la ressource dynamique du développement et de la créativité du média continental de la Chine. 


\section{Internationalisation, modernisation et régionalisation, un processus d'unification parallèle}

Le processus d'internationalisation, de modernisation et de régionalisation est un processus parallèle sans contradiction et favorable réciproquement. L'internationalisation de l'enseignement des médias est fondamentalement la réalisation de la modernisation: c'est un processus pour augmenter continuellement le degré de modernisation par l'apprentissage des expériences avancées d'éducation internationale. Pour accélérer la modernisation de l'enseignement des médias, on doit ouvrir la porte nationale, importer et exporter, différencier en comparant et prendre des expériences en différenciant. Donc, l'internationalisation de l'enseignement des médias est née avec la modernisation, on doit envisager la société internationale, renforcer la communication et la coopération internationales de l'enseignement des médias. en accélérer constamment l'internationalisation pour augmenter son degré de modernisation. Parallèlement. la modernisation est essentielle pour réaliser l'internationalisation, le processus d'internationalisation sera retardé si sont absents la pensée, le contenu et les méthodes modernes d'enseignement.

La relation de ces trois termes est dialectique. D'un côté, le processus d'internationalisation et de modernisation signifie lui-même la réforme et l'amélioration du régime et du procès d'opérations anciennes, c'est un changement de tradition : d'un autre côté, l'internationalisation et la modernisation ne signifient point «l'occidentalisation intégrale» ou "l'intégration internationale». elles sont nées et développées d'une culture autochtone : la régionalisation est une partie indispensable. De même, l'internationalisation et la modernisation de l'enseignement des médias sera définitivement effectuées par la régionalisation. Mais la régionalisation n'exclut pas l'internationalisation et la modernisation, elle nous impose seulement de considérer la situation nationale concrète et les différences locales en même temps que de stimuler l'internationalisation et la modernisation de renseignement des médias, pour se conformer aux besoins locaux, C'est-à-dire pour établir le système d'enseignement des médias qui possède lu spécificité chinoise et s'accorde avec la situation chinoise. 